\title{
Intramolecular Light-Driven Accumulation of Reduction Equivalents by Proton-Coupled Electron Transfer
}

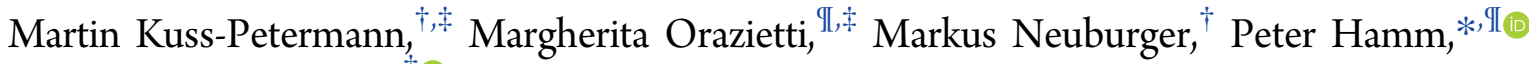 \\ and Oliver S. Wenger* ${ }^{*}+0$
}

${ }^{\dagger}$ Department of Chemistry, University of Basel, St. Johanns-Ring 19, 4056 Basel, Switzerland

${ }^{I}$ Department of Chemistry, University of Zurich, Winterthurerstrasse 190, 8057 Zurich, Switzerland

\section{Supporting Information}

ABSTRACT: The photochemistry of a molecular pentad composed of a central anthraquinone (AQ) acceptor flanked by two $\mathrm{Ru}(\mathrm{bpy})_{3}{ }^{2+}$ photosensitizers and two peripheral triarylamine (TAA) donors was investigated by transient IR and UV-vis spectroscopies in the presence of $0.2 \mathrm{M} p$ toluenesulfonic acid $(\mathrm{TsOH})$ in deaerated acetonitrile. In $\sim 15 \%$ of all excited pentad molecules, AQ is converted to its hydroquinone form $\left(\mathrm{AQH}_{2}\right)$ via reversible intramolecular electron transfer from the two TAA units $(\tau=65 \mathrm{ps})$, followed by intermolecular proton transfer from $\mathrm{TsOH}(\tau \approx 3 \mathrm{~ns}$ for the first step). Although the light-driven accumulation of reduction equivalents occurs through a sequence of electron and proton transfer steps, the resulting photoproduct decays via concerted PCET $(\tau=4.7 \mu \mathrm{s})$ with an $\mathrm{H} / \mathrm{D}$ kinetic isotope effect of $1.4 \pm 0.2$. Moreover, the reoxidation of $\mathrm{AQH}_{2}$ seems to take place via a double electron transfer step involving both $\mathrm{TAA}^{+}$units rather than sequential single electron transfer events. Thus, the overall charge-recombination reaction seems to involve a concerted proton-coupled two-electron oxidation of $\mathrm{AQH}_{2}$. The comparison of experimental data obtained in neat acetonitrile with data from acidic solutions suggests that the inverted driving-force effect can play a crucial role for obtaining long-lived photoproducts resulting from multiphoton, multielectron processes. Our pentad provides the first example of light-driven accumulation of reduction equivalents stabilized by PCET in artificial molecular systems without sacrificial reagents. Our study provides fundamental insight into how light-driven multielectron redox chemistry, for example the reduction of $\mathrm{CO}_{2}$ or the oxidation of $\mathrm{H}_{2} \mathrm{O}$, can potentially be performed without sacrificial reagents.

\section{INTRODUCTION}

Photoinduced electron transfer in donor-acceptor compounds commonly leads to the formation of electron-hole pairs, and in principle such charge-separated states are interesting for solar energy conversion because they resemble the primary photoproducts of photosystem II. ${ }^{1}$ However, in natural photosynthesis multiple redox equivalents must be accumulated transiently before they can be used for the actual water oxidation, hydrogen evolution, or $\mathrm{CO}_{2}$ reduction steps, ${ }^{2}$ because these are evidently multielectron redox transformations. Light-driven accumulation of redox equivalents is very tricky to perform in simple artificial systems, because once primary charge-separation has occurred, charge-recombination and other unproductive reactions compete very efficiently with charge accumulation. ${ }^{3,4}$ Nature stabilizes the intermediate redox products through proton-coupled electron transfer (PCET), ${ }^{5}$ for example in the oxygen-evolving $\mathrm{Mn}_{4} \mathrm{Ca}$ complex in which oxidation steps of the Kok cycle are coupled to deprotonation, ${ }^{2}$ or in the two-electron reduction of plastoquinone that is coupled to protonation. ${ }^{6}$ It seems attractive to exploit PCET for the light-driven accumulation of redox equivalents in artificial donor-acceptor compounds, but until now this has not been accomplished without sacrificial reagents.

When using sacrificial donors or acceptors, light-driven accumulation of redox equivalents is readily achievable because under these conditions the above-mentioned detrimental secondary reactions are largely suppressed. ${ }^{7-17}$ However, sustainable solar energy conversion will not be possible with this approach, and hence it is desirable to explore the basic principles of light-driven accumulation of redox equivalents without sacrificial reagents. Currently, there exist only a handful of molecular systems that are able to exhibit light-driven accumulation of redox equivalents under this restriction. Two early systems exhibited very short-lived doubly reduced species $(\tau \leq 5 \mathrm{~ns}),{ }^{18,19}$ and a third system relied on the use of $\mathrm{TiO}_{2}$ nanoparticles. $^{20,21}$ A related nanoparticle approach turned out to be useful for multielectron transfer in other systems, ${ }^{22}$ and nanoparticle or quantum dot based systems are of course generally promising for accumulation of redox equivalents. ${ }^{23,24}$

Received: February 15, 2017

Published: March 31, 2017 
Scheme 1. Molecular Structures of Pentad I, Triad II, and TAA-ref ${ }^{a}$

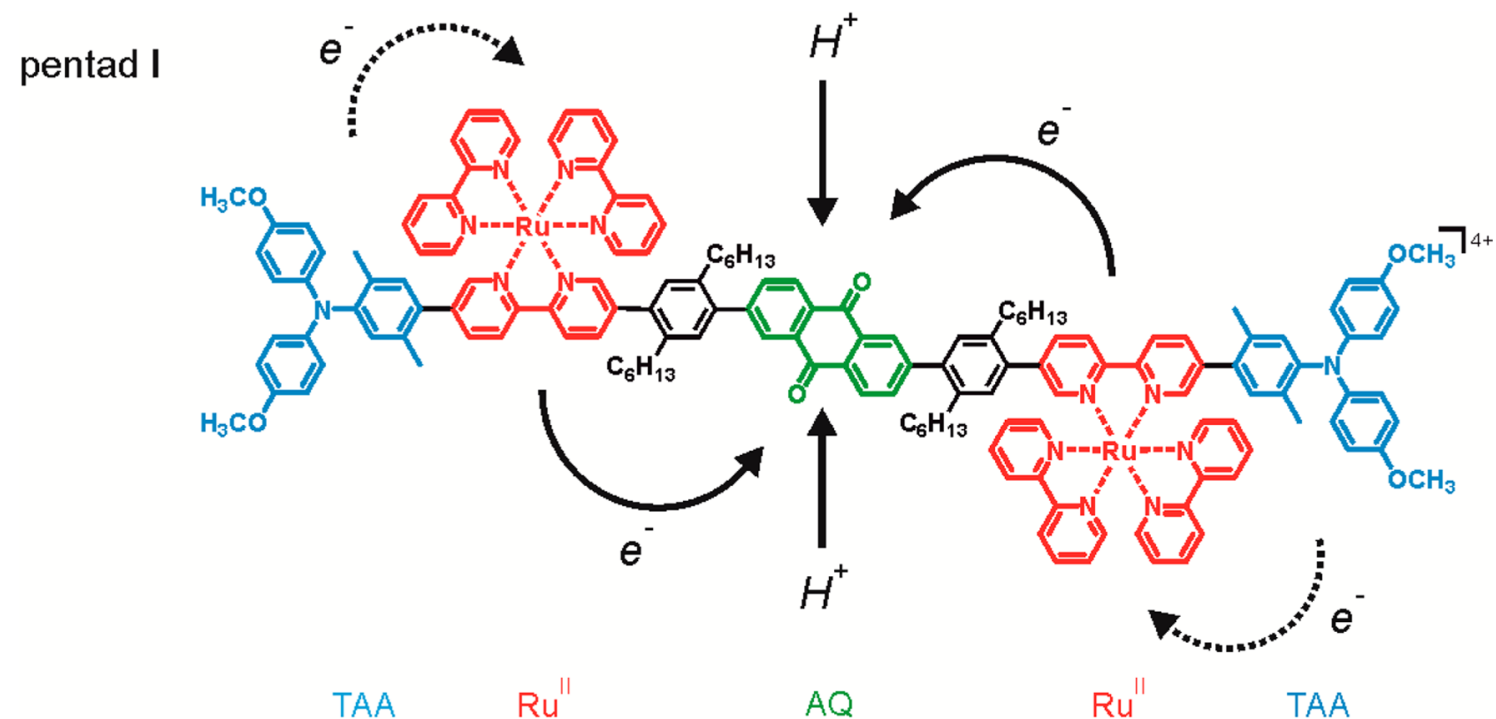

triad II

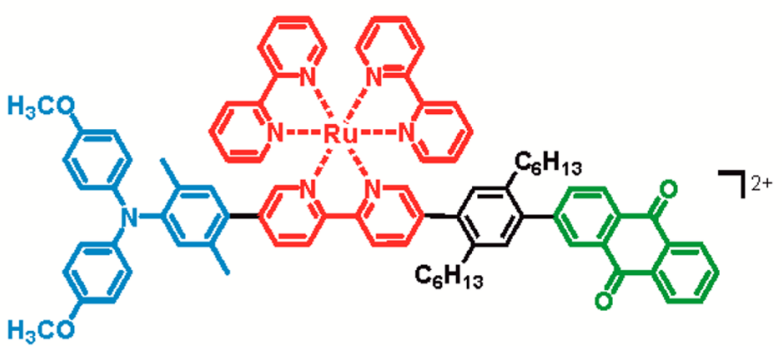

TAA-ref

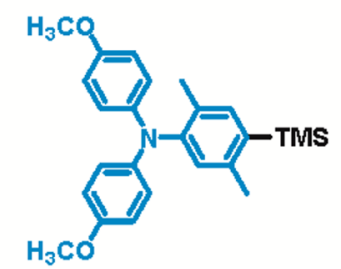

${ }^{a_{T}}$ The PCET chemistry expected for pentad $\mathbf{I}$ is illustrated.

We recently communicated the first purely molecular system (pentad I, Scheme 1) in which long-lived electron accumulation was possible without sacrificial reagents. ${ }^{25}$ Following excitation of the two $\mathrm{Ru}(\mathrm{II})$ chromophores, a doubly reduced anthraquinone species $\left(\mathrm{AQ}^{2-}\right)$ was observable and exhibited a lifetime of $870 \mathrm{~ns}$ in neat deaerated $\mathrm{CD}_{3} \mathrm{CN}$ at $20{ }^{\circ} \mathrm{C}$.

In this work, we explored pentad $\mathbf{I}$ in acetonitrile in the presence of excess $p$-toluenesulfonic acid $(\mathrm{TsOH})$, anticipating that the hydroquinone species $\left(\mathrm{AQH}_{2}\right)$ would be accessible under these conditions. Related prior work on single electron transfer with compounds similar to triad II (Scheme 1) provided evidence for the formation of semiquinones $\left(\mathrm{AQH}{ }^{\circ}\right),{ }^{26,27}$ and hence it seemed plausible that $\mathrm{AQH}_{2}$ could indeed form in pentad $\mathrm{I}$. We were interested to explore whether $\mathrm{AQH}_{2}$ would form through a concerted or a stepwise PCET mechanism and whether the $\mathrm{AQH}_{2}$ photoproduct would be even longer lived than the dianion species in neat $\mathrm{CD}_{3} \mathrm{CN}^{28,29}$ We find that the light-induced accumulation of reduction equivalents is a stepwise process involving consecutive electron and proton transfer events, but the reverse (thermal) chargerecombination seems to involve a concerted proton-coupled two-electron oxidation of $\mathrm{AQH}_{2}$. The inverted driving-force effect seems to play a crucial role for the lifetimes of the different doubly reduced photoproducts observed in neat acetonitrile and in the presence of acid.

The reduction of AQ in pentad I resembles the reduction of plastoquinone to plastoquinol in natural photosynthesis, in that multiple light-driven electron transfer reactions are coupled to two proton transfer events. ${ }^{6}$ Importantly, the overall reaction relies entirely on reversible redox reactions and the input of visible light. The anthraquinone-dihydroxyanthracene redox couple is used for the industrial synthesis of $\mathrm{H}_{2} \mathrm{O}_{2}$ from molecular oxygen, and consequently it seemed all the more relevant to explore the possibility of reversible two-electron, two-proton-coupled photochemistry in pentad $\mathbf{I}$.

\section{RESULTS AND DISCUSSION}

In the X-ray crystal structure of triad II (Figure 1), the centerto-center distance between the TAA donor and the AQ acceptor is $22.0 \AA$. TAA exhibits its common propeller-shaped structure, which is responsible for the relatively low basicity of triarylamines, and this is important for our spectroscopic studies performed in the presence of $\mathrm{TsOH}$. The dihedral angles between the central bpy ligand and its adjacent substituted phenylene bridging units $\left(44.6^{\circ}, 55.2^{\circ}\right)$ are in the typical range for $p$-xylenes. ${ }^{30}$ The structures of one of the methoxyphenyl groups, as well as that of cocrystallized solvent molecules (toluene, $\mathrm{CHCl}_{3}$ ) are disordered. Crystals of pentad I could not be obtained.

Infrared spectro-electrochemistry of isolated AQ in $\mathrm{CD}_{3} \mathrm{CN}$ at $20{ }^{\circ} \mathrm{C}$ provided the difference spectra in Figure 2a. The black trace was recorded in neat $\mathrm{CD}_{3} \mathrm{CN}$ at a potential of $-1.45 \mathrm{~V}$ vs $\mathrm{Fc}^{+/ 0}$, and consequently was attributed to $\mathrm{AQ}^{-31}$. When a potential of $-2.05 \mathrm{~V}$ vs $\mathrm{Fc}^{+/ 0}$ was applied, the formation of $\mathrm{AQ}^{2-}$ in neat $\mathrm{CD}_{3} \mathrm{CN}$ resulted in the red trace. In the presence

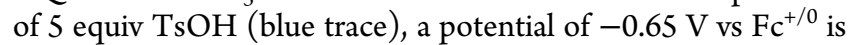
sufficient to lead to the formation of the $2 \mathrm{e}^{-} / 2 \mathrm{H}^{+}$reduction product $\mathrm{AQH}_{2}$ (the semiquinone, $\mathrm{AQH}{ }^{\bullet}$, cannot be isolated in 


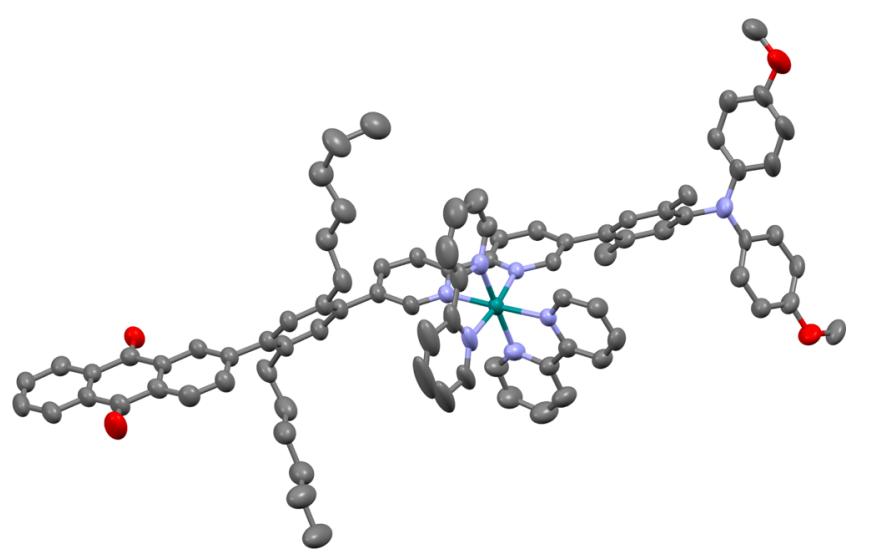

Figure 1. X-ray crystal structure of triad II with thermal ellipsoids at the $50 \%$ probability level. Hydrogen atoms, counteranions, and solvent molecules have been omitted.

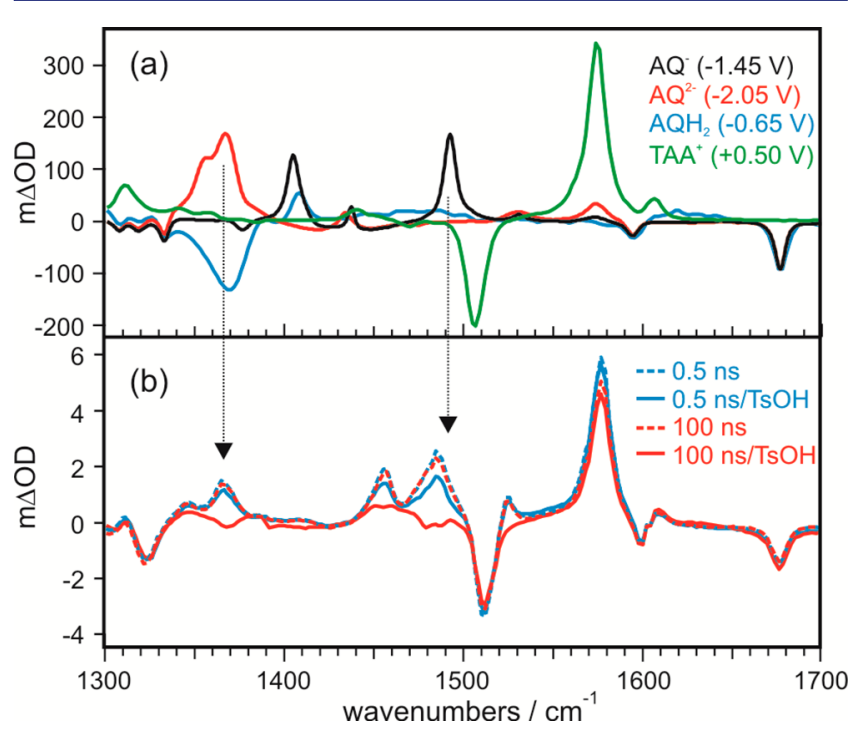

Figure 2. (a) IR difference spectra measured after electrochemical reduction of $\mathrm{AQ}(5 \mathrm{mM})$ to $\mathrm{AQ}^{-}$(black) and $\mathrm{AQ}^{2-}$ (red) and oxidation of TAA $(5 \mathrm{mM})$ to $\mathrm{TAA}^{+}$(using the TAA-ref compound from Scheme 1) in neat $\mathrm{CD}_{3} \mathrm{CN}$ (green). The difference spectrum of $\mathrm{AQH}_{2}$ (blue) was obtained after electrochemical reduction of $\mathrm{AQ}$ in the presence of 5 equiv $\mathrm{TsOH}$ in $\mathrm{CD}_{3} \mathrm{CN}$. The spectra measured prior to applying any potential served as a baseline. The blue spectrum was scaled to match the change in optical density at $1677 \mathrm{~cm}^{-1}$ in the black and red spectra. (b) Comparison of the transient IR spectra of pentad $\mathrm{I}$ in neat $\mathrm{CD}_{3} \mathrm{CN}$ (dashed lines) and in the presence of $0.2 \mathrm{M} \mathrm{TsOH}$ (solid lines). The spectra were recorded $0.5 \mathrm{~ns}$ (blue traces) and 100 ns (red traces) after excitation with 100 fs laser pulses $(2 \mu \mathrm{J})$ at 415 $\mathrm{nm}$.

the presence of protons due to potential inversions). Each one of these spectra exhibits bleach signals at 1332, 1594, and 1677 $\mathrm{cm}^{-1}$, originating from the depletion of AQ. Comparison of the two difference spectra recorded in absence of acid in Figure 2a (black and red traces) shows that the most diagnostic feature of $\mathrm{AQ}^{2-}$ is an IR absorption at $1366 \mathrm{~cm}^{-1}$ (marked by the left arrow in Figure 2), in line with prior reports. ${ }^{32,33}$ In contrast, $\mathrm{AQH}_{2}$ lacks similarly strong IR absorptions (blue trace). Aside from a bleach at $1370 \mathrm{~cm}^{-1}$ due to the deprotonation of $\mathrm{TsOH}$ (Figure S1), the only prominent features in the blue spectrum from Figure 2a is a band at $1407 \mathrm{~cm}^{-1}$, which is attributed to $\mathrm{AQH}_{2}$, but which is much weaker than the $1366 \mathrm{~cm}^{-1}$ marker mode of $\mathrm{AQ}^{2-}$. The IR difference spectrum resulting from

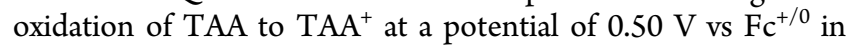
neat $\mathrm{CD}_{3} \mathrm{CN}$ is shown as a green trace in Figure 2a. The spectrum of $\mathrm{TAA}^{+}$is mainly composed of a bleach at 1506 $\mathrm{cm}^{-1}$ and a signal at $1575 \mathrm{~cm}^{-1}$.

Following excitation of $1 \mathrm{mM}$ pentad $\mathrm{I}$ in neat $\mathrm{CD}_{3} \mathrm{CN}$ with a femtosecond laser pulse at $415 \mathrm{~nm}$, the transient IR spectra in Figure $2 \mathrm{~b}$ were detected, shown here for delay times of 0.5 and $100 \mathrm{~ns}$ (blue dotted and red dotted lines, respectively). The comparison to the electrochemically induced difference spectra shown in Figure 2a allows us to assign these spectra. Most importantly, an absorption band at $1366 \mathrm{~cm}^{-1}$ is found in the transient spectra, similar to the $\mathrm{AQ}^{2-}$ spectrum in Figure 2a (red line), from which we had concluded in ref 25 that $A Q^{2-}$ is indeed formed in pentad I. The lack of that band in the triad II as well as its quadratic dependence on laser excitation power, indicating two absorbed photons, supported this assignment. We had estimated that under the conditions used to record the data in Figure $2 \mathrm{~b}, 15 \%$ of the excited pentad molecules exhibited electron accumulation on $\mathrm{AQ}$ to form $\mathrm{AQ}^{2-}$. The remaining $85 \%$ underwent only single electron transfer to result in $\mathrm{AQ}^{-}$, the latter of which being identified by a marker mode at $1492 \mathrm{~cm}^{-1}$ (see right arrow Figure 2, the spectrum of the reduced pentad I shows an additional band at $\sim 1460 \mathrm{~cm}^{-1}$ that is not present in $\mathrm{AQ}^{-}$; we assume that this band splits off from the $1492 \mathrm{~cm}^{-1}$ band due to the lowering of the symmetry of AQ in the pentad as compared to the isolated AQ). Both singly and doubly reduced states are formed in a few 10 s of ps and live for almost $1 \mu \mathrm{s}$, hence the transient spectra in absence of $\mathrm{TsOH}$ are practically identical after 0.5 and $100 \mathrm{~ns}$ (dotted traces in Figure 2b).

In the presence of $0.2 \mathrm{M} \mathrm{TsOH}$ in $\mathrm{CD}_{3} \mathrm{CN}$, the transient IR spectra shown in Figure $2 \mathrm{~b}$ (solid lines) are obtained. After 0.5 ns (blue solid line), the diagnostic absorption bands at 1366 and at $1485 \mathrm{~cm}^{-1}$ (slightly shifted from the corresponding band in Figure $2 \mathrm{a}$ ) are readily detectable, indicating that both $\mathrm{AQ}^{2-}$ and $\mathrm{AQ}^{-}$initially form also under acidic conditions. However, 100 ns after excitation (red solid line) both bands have disappeared while other bands, i.e., the $\mathrm{TAA}^{+}$-related bands at 1575 and at $1510 \mathrm{~cm}^{-1}$, as well as bleach contributions from $A Q$ at 1324,1598 , and $1675 \mathrm{~cm}^{-1}$ remain. In the presence of acid, the $\mathrm{AQ}^{-}$and $\mathrm{AQ}^{2-}$ species obviously disappear very rapidly. Because the oxidation product $\mathrm{TAA}^{+}$persists for much longer and $\mathrm{AQ}$ is not yet reoxidized, there must be long-lived reduction products other than $\mathrm{AQ}^{-}$and $\mathrm{AQ}^{2-}$. It seems plausible that these reduction products are protonated forms thereof. The bleach at $1370 \mathrm{~cm}^{-1}$, reflecting the deprotonation of $\mathrm{TsOH}$, supports that assumption.

Isolated $\mathrm{AQH}_{2}$ only shows an absorption band at $1407 \mathrm{~cm}^{-1}$ (blue trace in Figure 2a), which is not detectable in the transient spectra of pentad I, presumably because it is a weak band. At the same time, $\mathrm{AQH}_{2}$ can be populated only to maximal $15 \%{ }^{25}$ Because the reference spectrum of the semiquinone $\mathrm{AQH}^{\bullet}$ is not accessible due to potential inversions (see comments above), we resort to quantum chemistry calculations performed on the B3LYP/6-311++G(3df,3pd) level of theory with the polarizable continuum model for acetonitrile (see Figure S2). ${ }^{34}$ The reliability of these calculations is validated for the $\mathrm{AQH}_{2} / \mathrm{AQ}$ difference spectrum, which is reproduced essentially quantitatively (compare blue trace in Figure 2a with Figure S2 and keeping in mind that the dominating bleach at $1370 \mathrm{~cm}^{-1}$ originates from the deprotonation of $\mathrm{TsOH})$. The $\mathrm{AQH}^{\bullet} / \mathrm{AQ}$ difference spectrum 
(red trace in Figure S2), on the other hand, contains only one significant marker mode at $\sim 1590 \mathrm{~cm}^{-1}$, which likely overlaps with the much stronger band of $\mathrm{TAA}^{+}$. Hence, unfortunately, we are blind to the protonated forms of the reduced anthraquinone by transient IR spectroscopy.

Kinetic traces of the signals related to $\mathrm{AQ}^{2-}$ and $\mathrm{AQ}^{-}$are shown in Figure $3 \mathrm{a}, \mathrm{b}$ as a function of acid concentration. In

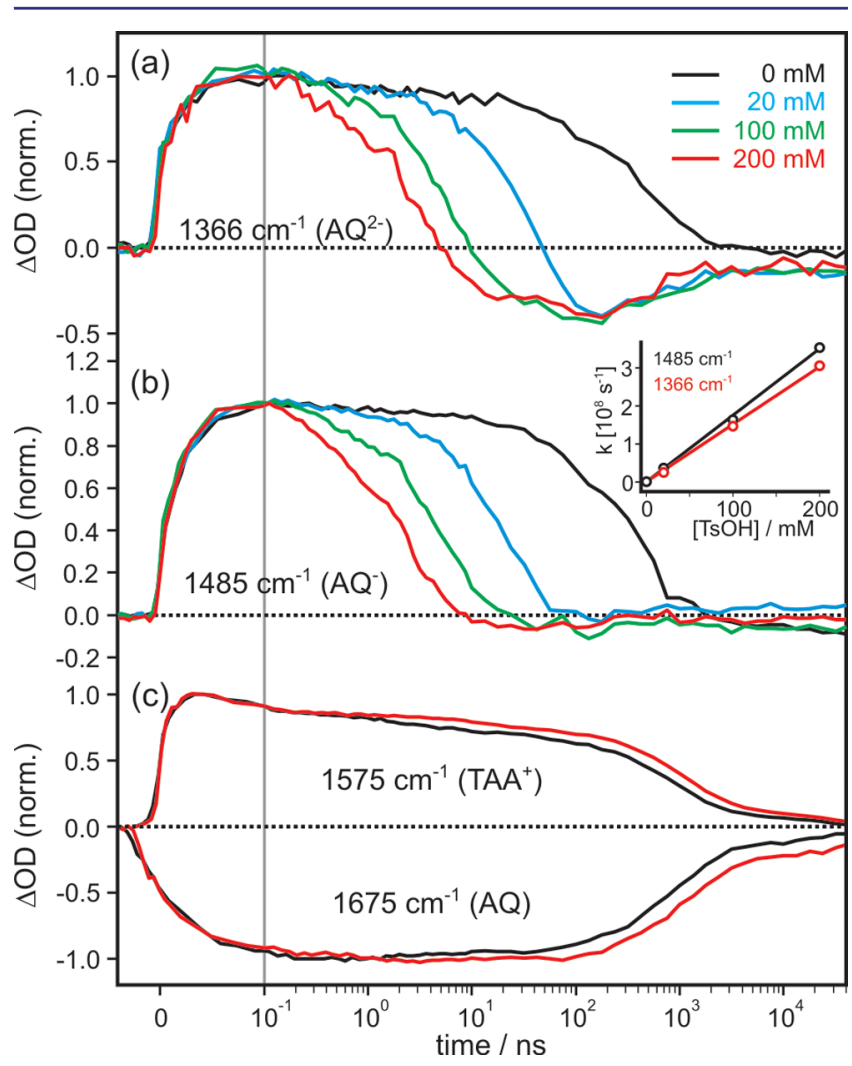

Figure 3. Normalized kinetics of different TRIR absorptions in the presence of increasing $\mathrm{TsOH}$ concentrations in $\mathrm{CD}_{3} \mathrm{CN}$. The solutions contained $1 \mathrm{mM}$ pentad $\mathrm{I}$ and were excited at $415 \mathrm{~nm}$ with laser pulses of $\sim 100 \mathrm{fs}$ duration (the rise-time of the signal is slower than the pulse duration due to the jitter in the synchronization of pump and probe lasers). ${ }^{25}$ Detection occurred by monitoring bands related to $\mathrm{AQ}^{2-}$ (a) and $\mathrm{AQ}^{-}(\mathrm{b})$. The inset shows the linear dependence of the decay kinetics at 1485 and $1366 \mathrm{~cm}^{-1}$ as a function of acid concentration. Panel $\mathrm{c}$ shows the normalized kinetics associated with the signal of $\mathrm{TAA}^{+}$and the ground state bleach of $\mathrm{AQ}$ in neat $\mathrm{CD}_{3} \mathrm{CN}$ (black lines) and in the presence of $200 \mathrm{mM}$ TsOH (red lines). Note that up to 0.1 $\mathrm{ns}$ the time scale is linear.

neat deaerated $\mathrm{CD}_{3} \mathrm{CN}, \mathrm{AQ}^{2-}$ and $\mathrm{AQ}^{-}$decay with lifetimes of 870 and 980 ns (black traces in Figure 3a,b), respectively. ${ }^{25}$ In the presence of $\mathrm{TsOH}$, the respective lifetimes shorten considerably (colored traces in Figure 3a,b), and there is a linear relationship between lifetimes and acid concentration (Figure $3 \mathrm{~b}$, inset), compatible with the transfer of a single proton. A protonation rate of $(1.5 \pm 0.2) \times 10^{9} \mathrm{M}^{-1} \mathrm{~s}^{-1}$ can be inferred from the linear fit, indicating a nearly diffusion controlled mechanism. ${ }^{35}$ With a single protonation step, both the $\mathrm{AQ}^{2-}$ (Figure 3a) and the $\mathrm{AQ}^{-}$(Figure 3b) marker modes will disappear. A hydroquinone anion species, $\mathrm{AQH}^{-}$, might transiently be formed from $\mathrm{AQ}^{2-}$ that then can be protonated a second time. We have no spectroscopic indication for a second protonation step; however, we expect it to happen on the same time scale as the first protonation step. The $\mathrm{p} K_{\mathrm{a}}$ of an acid is related to its deprotonation rate, which may vary over many orders of magnitude, whereas the protonation rate of the conjugated base is diffusion controlled and thus essentially a constant. ${ }^{36}$ That is, as long as the second protonation step is thermodynamically possible based on the acidity constants, it will happen on essentially the same time scale as the first protonation step. The $\mathrm{pK}$ alue of $\mathrm{TsOH}$ in $\mathrm{CH}_{3} \mathrm{CN}$ is 8.6. ${ }^{37}$ For the reduced $\mathrm{AQ}$ species, only $\mathrm{p} K_{\mathrm{a}}$ values for aqueous solvent seem to be known, and it is difficult to use these as a basis for estimation of the $\mathrm{pK}$ values in $\mathrm{CH}_{3} \mathrm{CN}$, due to the hydrogen-bonding nature of water. Consequently, we use reported values for 1,4-benzoquinone (BQ) in DMSO to estimate the acidity of the relevant reduced AQ species in acetonitrile. $^{38}$ Following a previously published procedure, ${ }^{37}$ one obtains $\mathrm{p} K_{\mathrm{a}}\left(\mathrm{BQH}^{-}\right)=35.2, \mathrm{p} K_{\mathrm{a}}\left(\mathrm{BQH}_{2}\right)=29.3$, and $\mathrm{p} K_{\mathrm{a}}\left(\mathrm{BQH}^{\bullet}\right)=21.5$. Assuming a similar $\mathrm{pK}_{\mathrm{a}}$ value for $\mathrm{AQH}_{2}$ as for $\mathrm{BQH}_{2}$ (29.3), the second protonation step is thus indeed expected to be exergonic by $\sim 1.2 \mathrm{eV}$. The kinetic traces at 1443 $\mathrm{cm}^{-1}$ (see Figure S3) indicate an additional kinetic process between $\sim 7-50$ ns at high acid concentrations. Because that process appears in essentially the same way in both the pentad I and the triad II, we conclude that it does not reflect the second protonation step. Tentatively, we assign it to a conformational change.

On a microsecond time scale, the AQ-bleach signal as well as the $\mathrm{TAA}^{+}$signal relax as well, indicating back-electron transfer (Figure $3 \mathrm{c}$ ). In the presence of protons, that decay is slightly slower. However, the decay occurs in a rather nonexponential manner with a tail extending beyond $40 \mu \mathrm{s}$ (the upper limit of our setup) that is strongly concentration dependent, indicating a bimolecular charge-recombination that separates reductive and oxidative equivalents on different molecules (Figure S7). Furthermore, we have seen that residual oxygen affects the decay time. We therefore will discuss the charge-recombination in more detail based on transient UV-vis experiments in the following, which can be performed at much lower concentrations (i.e., $20 \mu \mathrm{M}$, as compared to $1 \mathrm{mM}$ for the transient IR experiments), and under better deaerated conditions (because no flow-cell is required). We will also see that these UV-vis experiments are complementary to transient IR spectroscopy, as they can indeed identify the hydroquinone $\left(\mathrm{AQH}_{2}\right)$ and discriminate it from the semiquinone $\left(\mathrm{AQH}^{\bullet}\right)$, whereas transient IR spectroscopy has been blind to these two species.

The transient UV-vis data in Figure 4a were obtained by exciting pentad I at $532 \mathrm{~nm}$ with pulses of $\sim 10 \mathrm{~ns}$ duration in deaerated $\mathrm{CH}_{3} \mathrm{CN}$ containing $0.2 \mathrm{M}$ TsOH. Using such a long laser pulse width, all relevant photoproducts are formed within the duration of the pulses (see above). Transient absorption decays were recorded in $5 \mathrm{~nm}$ intervals between 320 and 800 $\mathrm{nm}$, using a detection time window of $40 \mu \mathrm{s}$. The resulting decay data (97 transients) were fitted globally to yield speciesassociated difference spectra (SADS). ${ }^{39}$ The best fit was obtained using a biexponential decay function with a very small offset $(\sim 1 \%)$, the latter reflecting residual signals with decay times longer than $40 \mu \mathrm{s}$ (related to the remaining bimolecular electron transfer at these low concentrations, see Figure S7). Analogous SADS fits to UV-vis transient absorption data for triad II are best fitted with a singleexponential decay function with a very small offset (Figure S8). Exemplary decay data and fits for pentad I and the triad II are in the Supporting Information (Figure S9). 


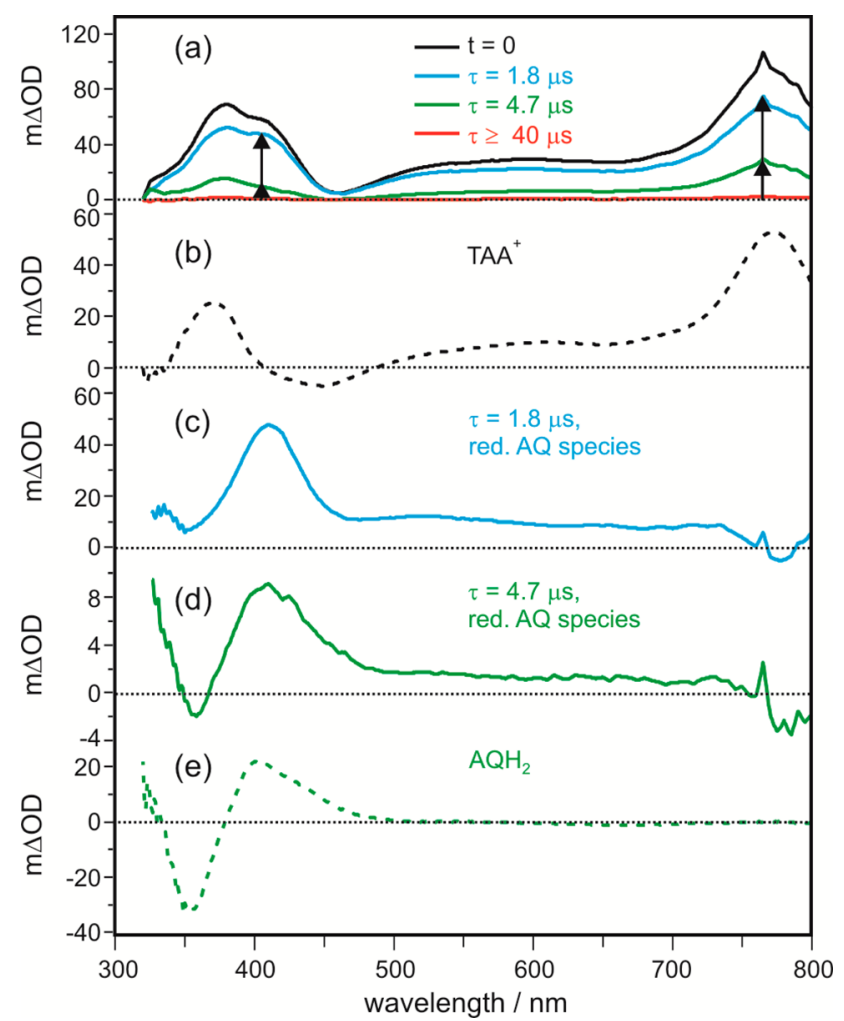

Figure 4. (a) Transient absorption data from a deaerated $\mathrm{CH}_{3} \mathrm{CN}$ solution of $20 \mu \mathrm{M}$ pentad I with $0.2 \mathrm{M} \mathrm{TsOH}$ at $20^{\circ} \mathrm{C}$. Excitation was at $532 \mathrm{~nm}$ with laser pulses of $\sim 10 \mathrm{~ns}$ duration. Black trace: Initial spectrum at $t=0$. Blue, green, red traces: SADS with the decay times indicated in the inset. The arrows at 407 and $770 \mathrm{~nm}$ illustrate that the $\Delta \mathrm{OD}_{407} / \Delta \mathrm{OD}_{770}$ ratio decreases from 1:1.5 in the blue trace to $1: 3.1$ in the green trace. (b) Differential absorption spectrum showing the spectral changes associated with oxidation of TAA in pentad I, obtained by spectro-electrochemistry in $\mathrm{CH}_{3} \mathrm{CN}$ (applied potential: $0.4{\mathrm{~V} \mathrm{vs} \mathrm{Fc}^{+/ 0}}^{+}$). (c) Contribution of reduced AQ species to the overall $1.8 \mu \mathrm{s}$-SADS, obtained by subtracting the appropriately scaled spectrum from (b) from the blue trace in panel a. (d) Contribution of reduced AQ species to the overall $4.7 \mu \mathrm{s}$-SADS, obtained by subtracting the appropriately scaled spectrum from (b) from the green trace in panel a. (e) Differential absorption spectrum for $\mathrm{AQH}_{2}$ obtained after applying a potential of $-1.4 \mathrm{~V} \mathrm{vs} \mathrm{Fc}^{+/ 0}$ to a solution containing $0.2 \mathrm{mM}$ pentad $\mathrm{I}, 4 \mathrm{mM}$ chloroacetic acid, and $0.1 \mathrm{M}$ $\mathrm{TBAPF}_{6}$ in $\mathrm{CH}_{3} \mathrm{CN}$; the spectrum obtained prior to applying any potential served as a baseline.

The black trace in Figure 4a represents the spectrum at $t=0$, which decays with components of $1.8 \mu$ s (blue trace) and $4.7 \mu \mathrm{s}$ (green trace). The transient difference spectra in Figure $4 \mathrm{a}$ are essentially linear combinations of the spectra of oxidation and reduction products. The oxidation product is $\mathrm{TAA}^{+}$in all cases, as seen readily from prominent absorptions at $\sim 770 \mathrm{~nm}^{40,41}$ The difference spectrum of $\mathrm{TAA}^{+}$in pentad $\mathbf{I}$, obtained by spectro-electrochemistry, is shown in Figure $4 \mathrm{~b}$. Reduced anthraquinones do not absorb significantly in the $600-800 \mathrm{~nm}$ spectral range, ${ }^{42,43}$ and consequently it is possible to scale the $\mathrm{TAA}^{+}$difference spectrum from Figure $4 \mathrm{~b}$ to match the $\Delta \mathrm{OD}$ values at $770 \mathrm{~nm}$ in the individual SADS from Figure 4a, in order to subtract the contribution of $\mathrm{TAA}^{+}$to these spectra. What remains are the contributions of the reduced AQ species to the individual SADS, and the resulting spectra are shown in Figure $4 \mathrm{c}(\tau=1.8 \mu \mathrm{s})$ and Figure $4 \mathrm{~d}(\tau=4.7 \mu \mathrm{s})$. The spectra in Figure $4 c / d$ are rather similar to one another, and with main absorption bands between 370 and $480 \mathrm{~nm}$ they are both compatible with the formation of either $\mathrm{AQH}^{\bullet}$ or $\mathrm{AQH}_{2}$, i.e., the semiquinone or hydroquinone forms. ${ }^{28,29} \mathrm{AQH}^{\bullet}$ and $\mathrm{AQH}_{2}$ have similar extinction coefficients at their absorption maxima in the blue/UV spectral range. Specifically, for $\mathrm{AQH}^{\bullet}$ in aqueous isopropanol/acetone mixture, $\varepsilon=8900 \mathrm{M}^{-1} \mathrm{~cm}^{-1}$ at $389 \mathrm{~nm},{ }^{44}$ whereas for $\mathrm{AQH}_{2}$ in ethanol/isopropyl alcohol the extinction coefficient at $382 \mathrm{~nm}$ is $\sim 7500 \mathrm{M}^{-1} \mathrm{~cm}^{-1} \cdot{ }^{45,46}$ Going back to the SADS in Figure 4a, we note that the ratio between $\Delta \mathrm{OD}$-values at $407 \mathrm{~nm}$ (i.e., near the maxima of the reduced AQ species in Figure 4c,d) and the $\Delta O D$ values at $770 \mathrm{~nm}$ (i.e., at the maximum of the $\mathrm{TAA}^{+}$absorption) is significantly different for the $1.8 \mu \mathrm{s}$ and the $4.7 \mu$ s components. Incidentally, $\mathrm{TAA}^{+}$does not contribute to the change in absorbance at 407 $\mathrm{nm}$ (Figure $4 \mathrm{~b}$ ), and consequently these ratios can be used to estimate the relative quantities of reduced AQ species and $\mathrm{TAA}^{+}$. From the blue trace in Figure $4 \mathrm{a}$ we extract a $\Delta \mathrm{OD}_{407} /$ $\Delta \mathrm{OD}_{770}$ ratio of $1: 1.5$, whereas in the green trace the respective ratio is 1:3.1 (see arrows in Figure 4a). Assuming the extinction coefficients of $\mathrm{AQH}^{\bullet}$ and $\mathrm{AQH}_{2}$ at $407 \mathrm{~nm}$ are indeed similar as noted above, the differences in $\Delta \mathrm{OD}_{407} / \Delta \mathrm{OD}_{770}$ ratios can then be explained by attributing the $1.8 \mu \mathrm{s}$ SADS to a $1: 1$ mixture of $\mathrm{AQH}^{\bullet}$ and $\mathrm{TAA}^{+}$, and by attributing the $4.7 \mu \mathrm{s}$ SADS to a 1:2 mixture of $\mathrm{AQH}_{2}$ and $\mathrm{TAA}^{+}$. In other words, the $1.8 \mu \mathrm{s}$ SADS corresponds to an ordinary electron-hole pair which is accessible after absorption of a single photon, whereas the $4.7 \mu$ s SADS reflects the charge-accumulated state comprised of a two-electron reduced $\mathrm{AQH}_{2}$ product combined with two one-electron oxidized species.

UV-vis spectro-electrochemical studies support this interpretation. The difference spectrum in Figure $4 \mathrm{e}$ was obtained after applying a potential of $-1.4 \mathrm{~V} \mathrm{vs} \mathrm{Fc}^{+/ 0}$ to a $0.2 \mathrm{mM}$ solution of pentad $\mathrm{I}$ in deaerated $\mathrm{CH}_{3} \mathrm{CN}$ with $4 \mathrm{mM}$ chloroacetic acid. This difference spectrum is in very good agreement with that of the AQ reduction product in Figure $4 \mathrm{~d}$. We note that the spectrum of the hydroquinone anion species $\left(\mathrm{AQH}^{-}\right)$exhibits an additional absorption band between 450 and $600 \mathrm{~nm},{ }^{42}$ hence it seems clear that the observed species in the $4.7 \mu$ s SADS does indeed contain the doubly protonated $\mathrm{AQH}_{2}$ product rather than $\mathrm{AQH}^{-}$. This is not surprising because $\mathrm{TsOH}$ is present in large excess and it is a much stronger acid than $\mathrm{AQH}_{2}$, as discussed above.

Based on the relative optical densities of the $\mathrm{TAA}^{+}$ absorptions at $770 \mathrm{~nm}$ in the blue and green traces of Figure $4 \mathrm{a}$, the relative amounts of $\mathrm{AQH}^{\bullet}$ and $\mathrm{AQH}_{2}$ can be estimated. Taking into account that the latter implies the formation of two $\mathrm{TAA}^{+}$units, we estimate that under the conditions used here (pulse energy of $15 \mathrm{~mJ}, P \approx 1.5 \mathrm{MW}$ ), roughly $15 \%$ of all photoexcited pentad molecules undergo double electron transfer to form $\mathrm{AQH}_{2}$ whereas the remaining $85 \%$ form the ordinary $\mathrm{AQH}^{\bullet}$ photoproduct resulting from single electron transfer (see Figure S10 for details). This estimate is in agreement with our prior study in neat $\mathrm{CD}_{3} \mathrm{CN}$, where we also estimated a ratio of $15 \%: 85 \%$ between $\mathrm{AQ}^{2-}$ and $\mathrm{AQ}^{-25}$.

As noted above, all photoproducts in Figure 4a form within the relatively long duration $(\sim 10 \mathrm{~ns})$ of the laser pulses used for this experiment, and consequently one merely detects their decay in UV-vis transient absorption. However, the TRIR studies reported above occurred with laser pulses of $\sim 100 \mathrm{fs}$ duration, and this large difference in excitation pulse widths is likely to lead to different reaction pathways for charge accumulation. In the TRIR experiment, both $\mathrm{Ru}(\mathrm{II})$ chromophores must be excited initially (Scheme 2, left), 
Scheme 2. Energy Level Diagram Established on the Basis of Electrochemical and UV-vis Data for Pentad $I^{a}$

(a) excitation with $\sim 100$ fs pulses

(b) excitation with $\sim 10$ ns pulses

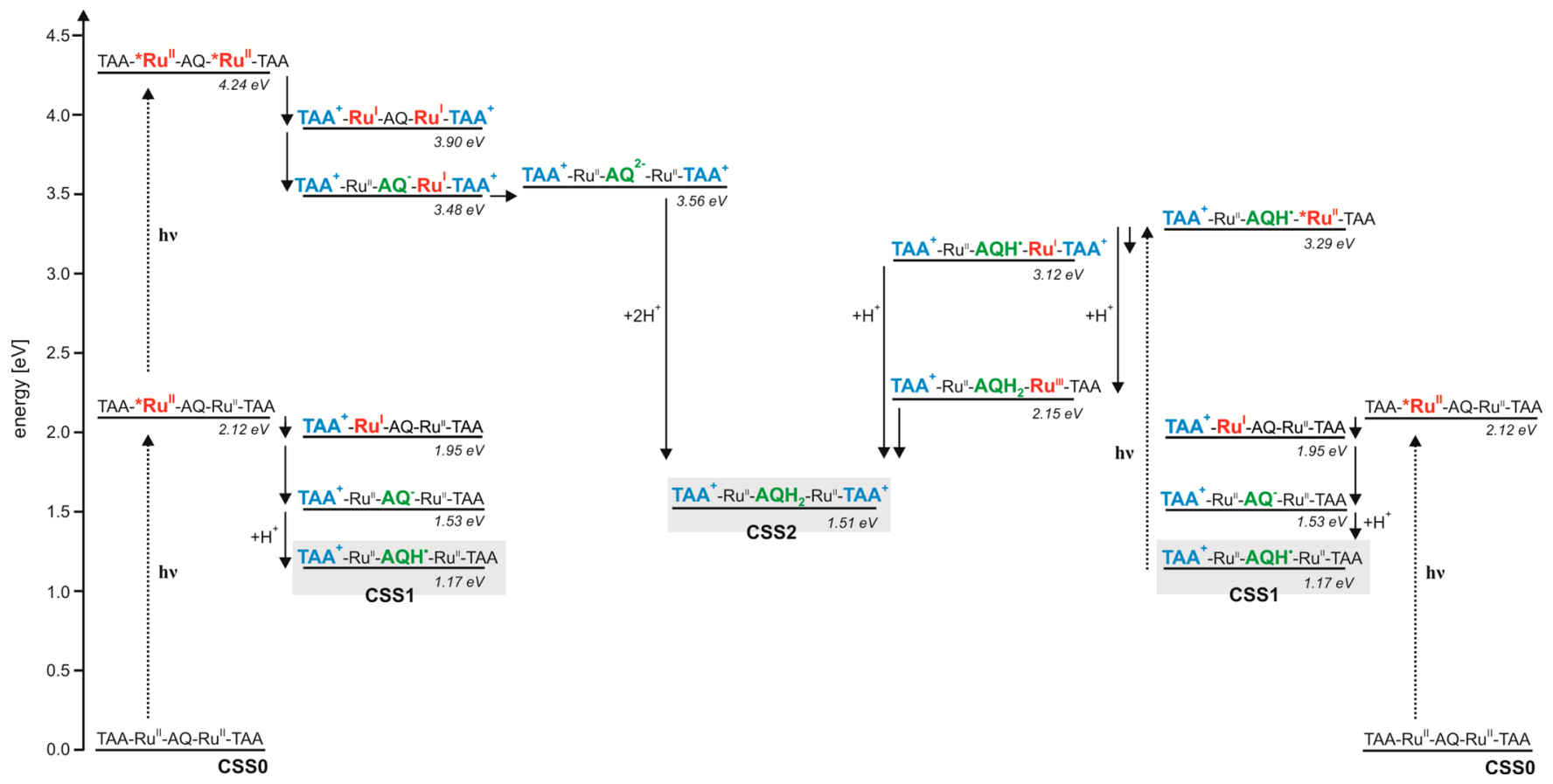

${ }^{a_{T}}$ The main reaction pathways leading to the accumulation of redox equivalents after excitation with $\sim 100 \mathrm{fs}$ pulses (left) and $\sim 10 \mathrm{~ns}$ pulses (right) are shown.

followed by electron transfer from TAA to the photoexcited complexes and subsequent reduction of $\mathrm{AQ}$. Although $\mathrm{AQ}^{2-}$ forms within $65 \mathrm{ps}^{25}$ the experiments performed in the presence of acid (Figures 2 and 3 ) show that protonation of $\mathrm{AQ}^{2-}$ occurs on a slower time scale $(\sim 3 \mathrm{~ns}$ at $0.2 \mathrm{M}$ TsOH for the first protonation step), indicating that $\mathrm{AQH}_{2}$ is formed via a sequence of electron and proton transfer events rather than via concerted PCET. Using the nanosecond pulses, a reaction sequence resembling more the Z-scheme of natural photosynthesis (Scheme 2, right) becomes viable. The formation of $\mathrm{TAA}^{+}$and $\mathrm{AQ}^{-}$requires only $50 \mathrm{ps}{ }^{25,47}$ and protonation of $\mathrm{AQ}^{-}$occurs with a time constant of $\sim 3$ ns (Figure 2). Consequently, when using pulses of $\sim 10$ ns duration, absorption of the second photon can occur after the formation of the primary $\mathrm{TAA}^{+} / \mathrm{AQH}^{\bullet}$ photoproduct (called CSS1 in Scheme 2), but ultimately the same final product comprised of two $\mathrm{TAA}^{+}$units and one $\mathrm{AQH}_{2}$ (CSS2) is accessible.

The lifetime of the $\mathrm{TAA}^{+} / \mathrm{AQH}^{\bullet}$ product in deaerated $\mathrm{CH}_{3} \mathrm{CN}$ is $1.8 \mu$ s (Figure $4 \mathrm{a}$ ), in line with prior experiments on compounds closely resembling triad II. ${ }^{27,47,48}$ The lifetime of the $\mathrm{AQH}_{2}$ photoproduct under identical conditions is $4.7 \mu \mathrm{s}$ (Figure $4 \mathrm{a}$ ), significantly longer than the lifetime of the $\mathrm{AQ}^{2-}$ species in neat $\mathrm{CD}_{3} \mathrm{CN}(870 \mathrm{~ns}){ }^{25}$ The energies of the individual states in Scheme 2 were determined based on the redox potentials of pentad $\mathbf{I}$ in the presence of $0.2 \mathrm{M} \mathrm{TsOH}$ (see Supporting Information for details; Figure S4).

When using deuterated $p$-toluenesulfonic acid (TsOD), the lifetimes of $\mathrm{AQD}^{\bullet}$ and $\mathrm{AQD}_{2}$ are 2.5 and $6.6 \mu \mathrm{s}$, respectively, under otherwise identical conditions (Figure S5). Thus, there is an H/D kinetic isotope effect (KIE) of $1.4 \pm 0.2$ for both recombination processes, compatible with concerted PCET to reform the AQ, TAA, and TsOD starting materials. ${ }^{49,50}$ In other words, intramolecular reverse electron transfer from
$\mathrm{AQH}^{\bullet}$ or $\mathrm{AQH}_{2}$ to $\mathrm{TAA}^{+}$occurs in concert with proton transfer to $\mathrm{TsO}^{-}$. However, so-called proton inventory experiments in which the H/D ratio was systematically varied in order to confirm the involvement of two protons in the case of $\mathrm{AQH}_{2}$ were inconclusive, ${ }^{51}$ due to the relatively weak KIE (Figure S6).

\section{SUMMARY AND CONCLUSIONS}

The formation of $\mathrm{AQ}^{2-}$ after absorption of two photons by the $\mathrm{Ru}(\mathrm{bpy})_{3}{ }^{2+}$ sensitizers has significant driving force (Scheme 2), and consequently there is no benefit from concerted PCET, and the overall process is a sequence of fast electron transfer (65 ps) and slower proton transfer events $(\sim 3 \mathrm{~ns}$ for the first step) when using excitation pulses of $\sim 100$ fs duration. With pulses of $\sim 10$ ns duration, $\mathrm{AQH}_{2}$ is also accessible via excitation of an intermediate with $\mathrm{AQH}^{\circ}$, because reduction and protonation to the semiquinone occurs with a time constant of $\sim 3$ ns. The resulting overall process then resembles the Zscheme of natural photosynthesis.

Given the large energetic stabilization of the $\mathrm{TAA}^{+}-\mathrm{AQH}_{2}-$ $\mathrm{TAA}^{+}$photoproduct relative to $\mathrm{TAA}^{+}-\mathrm{AQ}^{2-}-\mathrm{TAA}^{+}$(difference of $2.05 \mathrm{eV}$, Scheme $2 \mathrm{a}$ ), the lifetime prolongation from $870 \mathrm{~ns}$ in neat deaerated acetonitrile to $4.7 \mu \mathrm{s}$ in the presence of excess $\mathrm{TsOH}$ is rather modest. It seems plausible that the photoproduct in neat $\mathrm{CH}_{3} \mathrm{CN}$ benefits from the inverted drivingforce effect, because single electron transfer from $\mathrm{AQ}^{2-}$ to $\mathrm{TAA}^{+}$(to result in the $\mathrm{TAA}^{+}-\mathrm{AQ}^{-}$-TAA state at $1.53 \mathrm{eV}$ ) is expected to be exergonic by $\sim 2 \mathrm{eV}$, and double electron transfer from $\mathrm{AQ}^{2-}$ to two $\mathrm{TAA}^{+}$units even liberates $3.56 \mathrm{eV}$ (Scheme 2a). For reference, in previously investigated compounds closely related to triad II, charge-recombination between $\mathrm{AQ}^{-}$and $\mathrm{TAA}^{+}$was exergonic by $\sim 1.5 \mathrm{eV}$ and occurred clearly in the inverted region. ${ }^{52,53}$ By contrast, in 
acidic $\mathrm{CH}_{3} \mathrm{CN}$ solution, oxidation of $\mathrm{AQH}_{2}$ by one $\mathrm{TAA}^{+}$ liberates only $0.34 \mathrm{eV}$ (see Figure S4 including comments and Table S1), and the proton-coupled (single) oxidation of $\mathrm{AQH}_{2}$ by $\mathrm{TAA}^{+}$(to result in the $\mathrm{TAA}^{+}$-AQH ${ }^{\circ}$-TAA (CSS1) state at $1.17 \mathrm{eV}$ ) only releases $\sim 0.3 \mathrm{eV}$, whereas proton-coupled (double) oxidation of $\mathrm{AQH}_{2}$ by two $\mathrm{TAA}^{+}$units liberates 1.51 $\mathrm{eV}$ (Scheme 2b). Thus, for both kinds of processes (single and double electron transfer) the decay of CSS2 is associated with at least $\sim 1.7 \mathrm{eV}$ less driving force in the presence of $\mathrm{TsOH}$ than in neat $\mathrm{CH}_{3} \mathrm{CN}$. This is in line with recombination processes taking place in the inverted (aprotic solution) and the normal regime (protic solution). This could explain why a state storing ca. $3.5 \mathrm{eV}(\tau=870 \mathrm{~ns})$ does not decay far more rapidly than a state storing only ca. $1.5 \mathrm{eV}(4.7 \mu \mathrm{s})$. Thus, the comparison of aprotic and protic solvent environments provides insight into the decay behavior of electron transfer products that store uncommonly large amounts of energy.

As noted above, $\mathrm{TAA}^{+}$is in principle thermodynamically competent to oxidize $\mathrm{AQH}_{2}$ to $\mathrm{AQH}_{2}^{+}\left(\Delta G_{\mathrm{ET}}^{0}=-0.34 \mathrm{eV}\right.$; Figure $\mathrm{S} 4$ ), but the experimentally determined rate for disappearance of $\mathrm{AQH}_{2}$ exhibits a significant H/D KIE (1.4 \pm 0.2 ). This observation is compatible with a PCET process, in which $\mathrm{AQH}_{2}$ is oxidized by $\mathrm{TAA}^{+}$and deprotonated by $\mathrm{TsO}^{-}$, respectively, in a concerted fashion. A sequence of proton and electron transfer steps is improbable because deprotonation of $\mathrm{AQH}_{2}$ to $\mathrm{AQH}^{-}$is expected to be endergonic by $\sim 1.2 \mathrm{eV}$ in the presence of $\mathrm{TsO}^{-}$in acetonitrile (based on $\mathrm{p} K_{\mathrm{a}}$ values for $\mathrm{TsOH}$ and 1,4-benzoquinone, as discussed above). In the simplest case, concerted PCET between $\mathrm{AQH}_{2}, 1$ equiv TAA ${ }^{+}$, and 1 equiv $\mathrm{TsO}^{-}$leads to the semiquinone species $\left(\mathrm{AQH}^{\bullet}\right)$, i.e., CSS1 at $1.17 \mathrm{eV}$. However, we note that there is no evidence for repopulation of the $\mathrm{TAA}^{+}-\mathrm{AQH}^{\bullet}$-TAA (CSS1) state from the $\mathrm{TAA}^{+}-\mathrm{AQH}_{2}-\mathrm{TAA}^{+}$(CSS2) state, indicating that the latter decays directly to the ground state. This would imply a concerted proton-coupled two-electron oxidation, a highly uncommon process in molecular donor-acceptor compounds.

A key feature of the system considered here is that the accumulation of reduction equivalents on AQ is an entirely reversible process without any sacrificial reagents, demonstrating that light-driven multielectron reactions that are coupled to proton transfer steps are possible in molecular systems devoid of sacrificial donors or acceptors. So far, pentad I is a unique example in this context, but the proof of principle is now made.

It is remarkable that a comparatively simple system such as pentad I is able to mimic the biologically relevant photodriven conversion of plastoquinone to plastoquinol, which relies on a much more complex enzyme machinery. With its relatively long lifetime $(4.7 \mu \mathrm{s})$, the dihydroxyanthracene $\left(\mathrm{AQH}_{2}\right)$ photoproduct can potentially be used as a temporary storage reservoir of reduction equivalents in order to drive slower secondary redox processes that subsequently lead to more stable reduction products. This would be similar to plastoquinol in photosystem II, which ultimately transfers its reduction equivalents to $\mathrm{NAD}^{+}$ and $\mathrm{CO}_{2}$.

\section{ASSOCIATED CONTENT}

\section{S Supporting Information}

The Supporting Information is available free of charge on the ACS Publications website at DOI: 10.1021/jacs.7b01605.

Syntheses and experimental methods, additional crystallographic, TRIR, transient UV-vis, and electrochemical data, as well as quantum chemical calculations (PDF)
Crystallographic data for triad II (CIF)

\section{AUTHOR INFORMATION}

\section{Corresponding Authors}

*peter.hamm@chem.uzh.ch

*oliver.wenger@unibas.ch

ORCID $\odot$

Peter Hamm: 0000-0003-1106-6032

Oliver S. Wenger: 0000-0002-0739-0553

\section{Author Contributions}

$\stackrel{\ddagger}{\ddagger}$ These two authors contributed equally.

\section{Notes}

The authors declare no competing financial interest.

\section{ACKNOWLEDGMENTS}

This research was funded by the Swiss National Science Foundation through grant number 200021-146231/1 and the NCCR Molecular Systems Engineering to O.S.W., and through grant number CRSII2_160801/1 to P.H., as well as by the URRP LightChEC of the University of Zürich to P.H.

\section{REFERENCES}

(1) Wasielewski, M. R. Chem. Rev. 1992, 92, 435-461.

(2) Kok, B.; Forbush, B.; McGloin, M. Photochem. Photobiol. 1970, 11, 457-475.

(3) Hammarström, L. Acc. Chem. Res. 2015, 48, 840-850.

(4) Kuss-Petermann, M.; Wenger, O. S. Helv. Chim. Acta 2017, 100, e1600283.

(5) Mayer, J. M.; Rhile, I. J.; Larsen, F. B.; Mader, E. A.; Markle, T. F.; DiPasquale, A. G. Photosynth. Res. 2006, 87, 3-20.

(6) Saito, K.; Rutherford, A. W.; Ishikita, H. Proc. Natl. Acad. Sci. U. S. A. 2013, 110, 954-959.

(7) Pellegrin, Y.; Odobel, F. Coord. Chem. Rev. 2011, 255, 25782593.

(8) Bonn, A. G.; Wenger, O. S. Chimia 2015, 69, 17-21.

(9) Konduri, R.; de Tacconi, N. R.; Rajeshwar, K.; MacDonnell, F. M. J. Am. Chem. Soc. 2004, 126, 11621-11629.

(10) Konduri, R.; Ye, H. W.; MacDonnell, F. M.; Serroni, S.; Campagna, S.; Rajeshwar, K. Angew. Chem., Int. Ed. 2002, 41, 31853187.

(11) Wouters, K. L.; de Tacconi, N. R.; Konduri, R.; Lezna, R. O.; MacDonnell, F. M. Photosynth. Res. 2006, 87, 41-55.

(12) Matt, B.; Fize, J.; Moussa, J.; Amouri, H.; Pereira, A.; Artero, V.; Izzet, G.; Proust, A. Energy Environ. Sci. 2013, 6, 1504-1508.

(13) Elvington, M.; Brewer, K. J. Inorg. Chem. 2006, 45, 5242-5244.

(14) Manbeck, G. F.; Brewer, K. J. Coord. Chem. Rev. 2013, 257, $1660-1675$.

(15) Molnar, S. M.; Nallas, G.; Bridgewater, J. S.; Brewer, K. J. J. Am. Chem. Soc. 1994, 116, 5206-5210.

(16) Bonn, A. G.; Wenger, O. S. Phys. Chem. Chem. Phys. 2015, 17, 24001-24010.

(17) Knör, G.; Vogler, A.; Roffia, S.; Paolucci, F.; Balzani, V. Chem. Commun. 1996, 1643-1644.

(18) O’Neil, M. P.; Niemczyk, M. P.; Svec, W. A.; Gosztola, D.; Gaines, G. L.; Wasielewski, M. R. Science 1992, 257, 63-65.

(19) Imahori, H.; Hasegawa, M.; Taniguchi, S.; Aoki, M.; Okada, T.; Sakata, Y. Chem. Lett. 1998, 27, 721-722.

(20) Karlsson, S.; Boixel, J.; Pellegrin, Y.; Blart, E.; Becker, H. C.; Odobel, F.; Hammarström, L. J. Am. Chem. Soc. 2010, 132, 1797717979.

(21) Karlsson, S.; Boixel, J.; Pellegrin, Y.; Blart, E.; Becker, H. C.; Odobel, F.; Hammarström, L. Faraday Discuss. 2012, 155, 233-252.

(22) Young, R. M.; Jensen, S. C.; Edme, K.; Wu, Y. L.; Krzyaniak, M. D.; Vermeulen, N. A.; Dale, E. J.; Stoddart, J. F.; Weiss, E. A.; 
Wasielewski, M. R.; Co, D. T. J. Am. Chem. Soc. 2016, 138, 61636170.

(23) Valdez, C. N.; Schimpf, A. M.; Gamelin, D. R.; Mayer, J. M. J. Am. Chem. Soc. 2016, 138, 1377-1385.

(24) Chen, J. Q.; Wu, K. F.; Rudshteyn, B.; Jia, Y. Y.; Ding, W. D.; Xie, Z. X.; Batista, V. S.; Lian, T. Q. J. Am. Chem. Soc. 2016, 138, 884892.

(25) Orazietti, M.; Kuss-Petermann, M.; Hamm, P.; Wenger, O. S. Angew. Chem., Int. Ed. 2016, 55, 9407-9410.

(26) Hankache, J.; Niemi, M.; Lemmetyinen, H.; Wenger, O. S. J. Phys. Chem. A 2012, 116, 8159-8168.

(27) Hankache, J.; Wenger, O. S. Chem. - Eur. J. 2012, 18, 64436447.

(28) Mayer, J. M. Annu. Rev. Phys. Chem. 2004, 55, 363-390.

(29) Weinberg, D. R; Gagliardi, C. J.; Hull, J. F.; Murphy, C. F.; Kent, C. A.; Westlake, B. C.; Paul, A.; Ess, D. H.; McCafferty, D. G.; Meyer, T. J. Chem. Rev. 2012, 112, 4016-4093.

(30) Hanss, D.; Walther, M. E.; Wenger, O. S. Coord. Chem. Rev. 2010, 254, 2584-2592.

(31) Quan, M.; Sanchez, D.; Wasylkiw, M. F.; Smith, D. K. J. Am. Chem. Soc. 2007, 129, 12847-12856.

(32) Cheng, W. X.; Jin, B. K.; Huang, P.; Cheng, L. J.; Zhang, S. Y.; Tian, Y. P. J. Phys. Chem. C 2013, 117, 3940-3948.

(33) Babaei, A.; Brooksby, P. A.; Flood, A.; McQuillan, A. J. Appl. Spectrosc. 2000, 54, 496-501.

(34) Frisch, M. J.; Trucks, G. W.; Schlegel, H. B.; Scuseria, G. E.; Robb, M. A.; Cheeseman, J. R.; Scalmani, G.; Barone, V.; Petersson, G. A.; Nakatsuji, H.; Li, X.; Caricato, M.; Marenich, A.; Bloino, J.; Janesko, B. G.; Gomperts, R.; Mennucci, B.; Hratchian, H. P.; Ortiz, J. V.; Izmaylov, A. F.; Sonnenberg, J. L.; Williams-Young, D.; Ding, F.; Lipparini, F.; Egidi, F.; Goings, J.; Peng, B.; Petrone, A.; Henderson, T.; Ranasinghe, D.; Zakrzewski, V. G.; Gao, J.; Rega, N.; Zheng, G.; Liang, W.; Hada, M.; Ehara, M.; Toyota, K.; Fukuda, R.; Hasegawa, J.; Ishida, M.; Nakajima, T.; Honda, Y.; Kitao, O.; Nakai, H.; Vreven, T.; Throssell, K.; Montgomery, J. A., Jr.; Peralta, J. E.; Oliaro, F.; Bearpark, M.; Heyd, J. J.; Brothers, E.; Kudin, N.; Staroverov, V. N.; Keith, T.; Kobayashi, R.; Normand, J.; Raghavachari, K.; Rendell, A.; Burant, J. C.; Iyengar, S. S.; Tomasi, J.; Cossi, M.; Millam, J. M.; Klene, M.; Adamo, C.; Cammi, R.; Ochterski, J. W.; Martin, R. L.; Morokuma, K.; Farkas, O.; Foresman, J. B.; Fox, D. J. Gaussian 09 Revision A.02; Gaussian, Inc.: Wallingford CT, 2016.

(35) Rosspeintner, A.; Angulo, G.; Vauthey, E. J. Am. Chem. Soc. 2014, 136, 2026-2032.

(36) Donten, M. L.; Hamm, P. J. Phys. Chem. Lett. 2011, 2, 16071611.

(37) Kütt, A.; Leito, I.; Kaljurand, I.; Soovali, L.; Vlasov, V. M.; Yagupolskii, L. M.; Koppel, I. A. J. Org. Chem. 2006, 71, 2829-2838. (38) Warren, J. J.; Tronic, T. A.; Mayer, J. M. Chem. Rev. 2010, 110, 6961-7001.

(39) van Stokkum, I. H. M.; Larsen, D. S.; van Grondelle, R. Biochim. Biophys. Acta, Bioenerg. 2004, 1657, 82-104.

(40) Sreenath, K.; Suneesh, C. V.; Gopidas, K. R.; Flowers, R. A. J. Phys. Chem. A 2009, 113, 6477-6483.

(41) Sreenath, K.; Thomas, T. G.; Gopidas, K. R. Org. Lett. 2011, 13, $1134-1137$.

(42) Babaei, A.; Connor, P. A.; McQuillan, A. J.; Umapathy, S. J. Chem. Educ. 1997, 74, 1200-1204.

(43) Pal, H.; Mukherjee, T.; Mittal, J. P. J. Chem. Soc., Faraday Trans. 1994, 90, 711-716.

(44) Rath, M. C.; Pal, H.; Mukherjee, T. Radiat. Phys. Chem. 1996, 47, 221-227.

(45) Tickle, K.; Wilkinson, F. Trans. Faraday Soc. 1965, 61, 19811990.

(46) Hamanoue, K.; Sawada, K.; Yokoyama, K.; Nakayama, T.; Hirase, S.; Teranishi, H. J. Photochem. 1986, 33, 99-111.

(47) Hankache, J.; Niemi, M.; Lemmetyinen, H.; Wenger, O. S. Inorg. Chem. 2012, 51, 6333-6344.

(48) Hankache, J.; Wenger, O. S. Chem. Commun. 2011, 47, 1014510147.
(49) Sjödin, M.; Styring, S.; Åkermark, B.; Sun, L. C.; Hammarström, L. J. Am. Chem. Soc. 2000, 122, 3932-3936.

(50) Moore, G. F.; Hambourger, M.; Gervaldo, M.; Poluektov, O. G.; Rajh, T.; Gust, D.; Moore, T. A.; Moore, A. L. J. Am. Chem. Soc. 2008, 130, 10466-10467.

(51) Matta, M. S.; Vo, D. T. J. Am. Chem. Soc. 1986, 108, 5316-5318.

(52) Kuss-Petermann, M.; Wenger, O. S. J. Am. Chem. Soc. 2016, 138, $1349-1358$.

(53) Kuss-Petermann, M.; Wenger, O. S. Angew. Chem., Int. Ed. 2016, $55,815-819$. 